PAPER • OPEN ACCESS

Interaction of inertia and cavitation in textured hydrodynamically lubricated bearing in very low sliding velocity considering slip

To cite this article: J Jamari et al 2020 J. Phys.: Conf. Ser. 1517012005

View the article online for updates and enhancements.

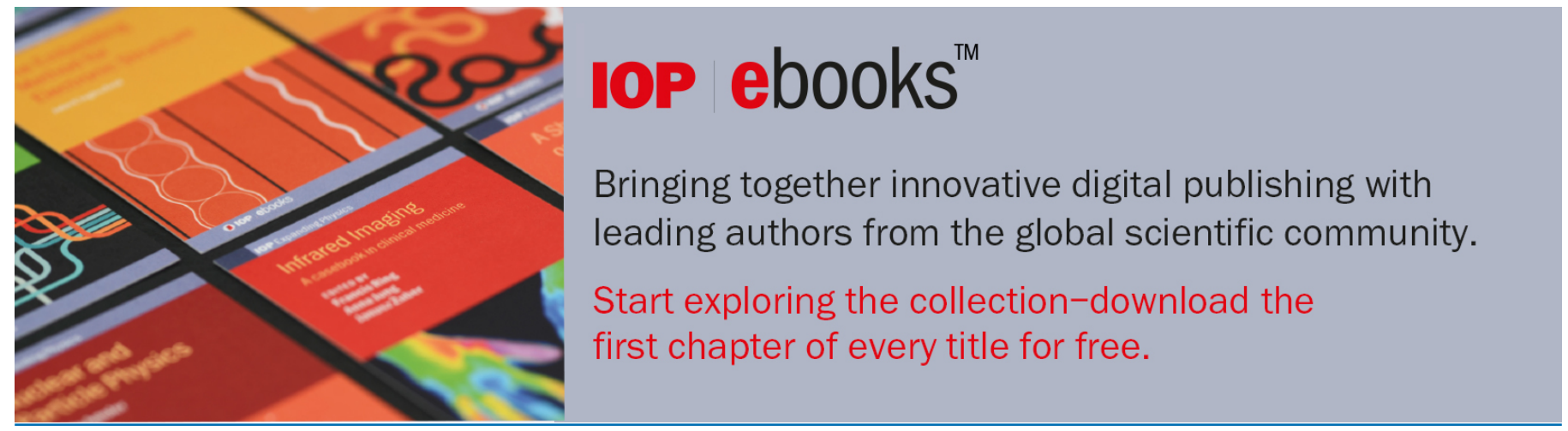




\title{
Interaction of inertia and cavitation in textured hydrodynamically lubricated bearing in very low sliding velocity considering slip
}

\author{
J Jamari $^{1}$, F Hilmy ${ }^{2}$, M Muchammad ${ }^{1,3}$, and M Tauviqirrahman ${ }^{1 *}$ \\ ${ }^{1}$ Laboratory for Engineering Design and Tribology, Mechanical Engineering \\ Department, Engineering Faculty, Diponegoro University, Semarang, Indonesia \\ ${ }^{2}$ Department of Mechanical Engineering, Faculty of Engineering, Universitas Tidar, \\ Magelang, Indonesia \\ ${ }^{3}$ Laboratory for Surface Technology and Tribology, Faculty of Engineering \\ Technology, University of Twente, Enschede, The Netherlands
}

*Email: mtauviq99@yahoo.com

\begin{abstract}
Surface texturing is becoming an effective way for increasing the performance of hydrodynamic bearings. However, introducing the texture on the lubricated sliding contact may lead to the presence of the inertia effect as well as the cavitation effect. In this study, the correlation between the inertia and the cavitation in textured bearing is investigated in terms of hydrodynamic pressure and load support. The Navier-Stokes equation based on CFD and modified Reynolds equation are applied. In addition, the use of slip condition and its effect on the performance of bearing is of particular interest. The simulations are conducted in very low sliding velocity to address the low inertia effect. The result shows that increasing inertia effect can increase the load support, but the cavitation effects is not found in the bearing pattern in which the inertia is not significant. The other interesting result concludes that slip reduces the load support, but on the other hand slip also increases the load support depending on the texture length.
\end{abstract}

\section{Introduction}

Textured surface have become an effective technique to improve the tribological performance of lubricated mechanical components. It is known that by introducing textured surfaces significantly can affect the load support of sliding bearings. In recent years, a great deal of effort has been addressed to consider the effect of the texture's geometric parameters on the lubrication performance. However, as is well known, when the texture is applied on the bearing, the inertia as well as the cavitation may exist and affect the lubrication performance.

Many researchers expressed that the inertia has a strong impact on the bearing performance either numerically $[1,2,4,5,7]$ or theoretically $[3,6,8]$. Based on the numerical simulations, Dobrica and Fillon [1] analysed the validity of Reynolds equation and proposed on how to take into account the inertia effect in modelling textured infinitely wide parallel sliders. These results showed that inertia has negative effects, reducing the load capacity of the slider.

However, texture on bearings may create significant issues such as the emergence of cavitation zone. Therefore, cavitation is strongly recommended to be considered in solving the hydrodynamic problem. 
Fowell et al. [9] observed the entrainment and inlet suction of two mechanisms of hydrodynamic lubrication in textured bearings. Their observation claimed that to get a great load support of pocketed bearings, the cavitation pressure should be as low as possible. On the other study, Shi and Ni [10] using the numerical method studied the effects of groove textures on fully lubricated sliding with cavitation. The simulation results showed that with the increase of cavitation pressure, the hydrodynamic pressure effect becomes more pronounced, while the friction force does not change very much.

Along with the development of technology, in order to get the best performance, the bearing surface can be modified by applying an artificial slip using a hydrophobic coating. The studies of the beneficial of slip have been investigated by Rao et al. [11], Zhang et al. [12], Tauviqirrahman et al. [13], Cheng and Ji [14]. Their results leads to the similarity of conclusions. All of them agree that the slip in certain pattern enables improvement of the tribological performance. In contrast, Zhang et al. [15] studied concluded that slip causes the pressure drop. Based on literature survey, one can find that most of studies have not considered the cavitation model in detail, and they do not consider the inertia term in their model.

Based on literature survey, one can find that the correlation between the inertia and the cavitation in lubricated sliding contact is still not clear, especially when the slip situation is applied. Thus, in this study, the interaction between inertia effect and cavitation condition considering slip is explored. In hydrodynamic problem of textured bearing is solved using modified Reynold equations (with slip) and compared to the CFD approach based on Navier-Stokes equation. In addition, the cavitation model is taken into account both for Reynolds equation and Navier-Stokes equation.

\section{Methods}

Figure 1 represents the illustration of a two-dimensional slider bearing model of single textured sider bearing. Slider bearing applied consists of two parallel walls with one as a stator (stationary wall) and another wall as the moving wall. The texture is on the stationary wall.

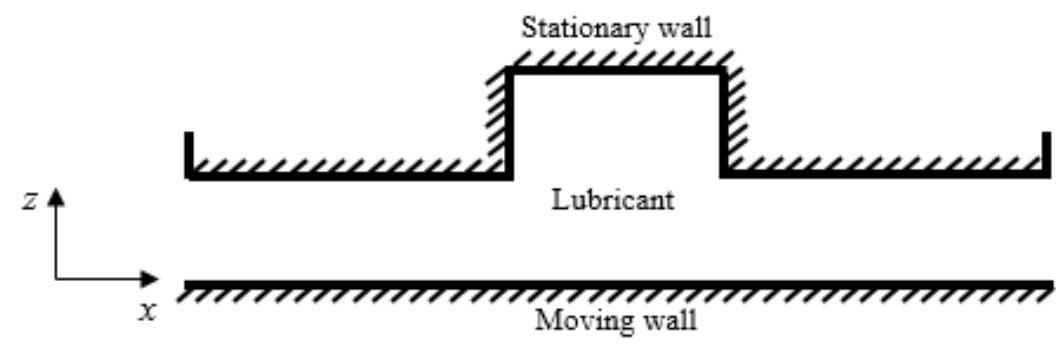

Figure 1. Model of two-dimensional slider bearing.

The main parameters of two-dimensional geometry model of slider bearing are follows: $B_{0}=2 \times 10^{-3}$ $\mathrm{m}$ (the length of bearing), $h_{0}=4 \times 10^{-6} \mathrm{~m}$ (the minimum film thickness), $h_{d}=4 \times 10^{-6} \mathrm{~m}$ (the texture depth), $a=0.75 \times 10^{-3} \mathrm{~m}$ (the inlet length), the value of $b$ (the texture length) is varied i.e. $0.25 \times 10^{-3}$, $0.50 \times 10^{-3}$ and $0.75 \times 10^{-3} \mathrm{~m}, c$ as the outlet length is also varied depending on the value of the texture length. The parameters are detailed on Fig. 2. Assuming the fluid material applied had a value of density $(\rho)$ and dynamic viscosity $(\mu)$ of $962 \mathrm{~kg} / \mathrm{m}^{3}$ and 0.013468 Pa.s. The velocity of moving wall $U$ is $0.1 \mathrm{~m} / \mathrm{s}$ is assumed as corresponding the Reynolds number $R e$ is 0.028 . In the following study, the coefficient of slip is 0.02 based on study of Muchammad et al. [16].

In the present study, to explore the correlation between the inertia, the cavitation, and the slip, three textured bearings are of main particular interest. First, the bearing with low texture length $(b=0.25$ $\mathrm{mm})$. Second, the bearing with medium texture length $(b=0.50 \mathrm{~mm})$ and third the bearing with high texture length $(b=0.75 \mathrm{~mm})$. The variation of $\mathrm{b}$ is aimed to alter the inertia effect. In the analysis, when slip is particular interest, the slip is implemented to all stationary surface including the texture cell as mentioned before. It should be noted that in this analysis, the inlet length $a$ is set to constant, i.e 0.75 
mm. As a consequently, when $b$ value is varied, the outlet length $c$ will change. For all following computation, the texture depth $h_{d}$ is set to $4 \mu \mathrm{m}$, which means that $h_{d}=h_{0}$.

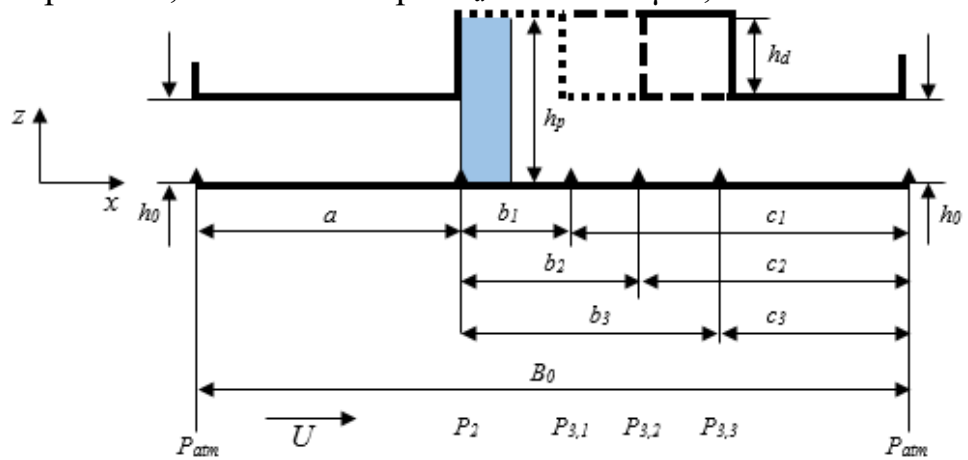

Figure 2. Geometry of twodimensional slider bearing.

To investigate the effect of inertia on hydrodynamic lubrication with boundary wall slip and considering cavitation, modifying Reynolds equation and numerical simulation by the computational fluid dynamics method are set.

\subsection{Reynold Equation}

An analytical equation based on first-order derivation of Reynolds approach is used in this present study. In this approach, the Reynolds equation is modified by including slip and cavitation. For detail, derivation of equation (1) the reader can refers to the published work of Muchammad et al. [16]. To resolve the problem using the governing equations employing an analytical solution for the slip case is:

$$
\begin{gathered}
P_{2}\left[\left(h_{p}^{3}+3 h_{p}^{3} K_{p}\right) \frac{a b+b c}{a b^{2}}+\left(\frac{h_{0}^{3}+3 h_{0}^{3} K_{0}}{a}\right)\right]=P_{a t m}\left[\left(h_{p}^{3}+3 h_{p}^{3} K_{p}\right) \frac{a b+b c}{a b^{2}}+\left(\frac{h_{0}^{3}+3 h_{0}^{3} K_{0}}{a}\right)\right] \\
-6 \mu U\left[\left(h_{p}+h_{p} K_{p}\right)-\left(h_{0}+h_{0} K_{0}\right)\right]
\end{gathered}
$$

In this study, the load support $W$ is also particular interest.

\subsection{Navier-Stokes Equation}

In the current study, the hydrodynamic lubrication is investigated by the Navier-Stokes equations using the commercial CFD software package FLUENT ${ }^{\circledR}$ which are determined over the domain applying a finite-volume method. The Navier-Stokes and the continuity equations can be represented, respectively, as [17]:

$$
\begin{gathered}
\rho\left(u \frac{\partial u}{\partial x}+v \frac{\partial u}{\partial y}+w \frac{\partial u}{\partial z}\right)-\mu\left(\frac{\partial^{2} u}{\partial x^{2}}+\frac{\partial^{2} u}{\partial y^{2}}+\frac{\partial^{2} u}{\partial z^{2}}\right)+\frac{\partial p}{\partial x}=0 \\
\rho\left(u \frac{\partial v}{\partial x}+v \frac{\partial v}{\partial y}+w \frac{\partial v}{\partial z}\right)-\mu\left(\frac{\partial^{2} v}{\partial x^{2}}+\frac{\partial^{2} v}{\partial y^{2}}+\frac{\partial^{2} v}{\partial z^{2}}\right)+\frac{\partial p}{\partial y}=0 \\
\rho\left(u \frac{\partial w}{\partial x}+v \frac{\partial w}{\partial y}+w \frac{\partial w}{\partial z}\right)-\mu\left(\frac{\partial^{2} w}{\partial x^{2}}+\frac{\partial^{2} w}{\partial y^{2}}+\frac{\partial^{2} w}{\partial z^{2}}\right)+\frac{\partial p}{\partial z}=0 \\
\frac{\partial u}{\partial x}+\frac{\partial v}{\partial y}+\frac{\partial w}{\partial z}=0
\end{gathered}
$$

\subsection{CFD Model}

The flow is assumed to be turbulent, incompressible, steady and using the Newtonian fluid. In the current study, the moving wall is varied by applying the full slip (boundary slip is applied on the entire surface 
of stationary wall including the surface of texture) and no slip boundary condition. The slippage boundary condition is shown in the Figure 3. At the inlet and outlet of the domain, the pressure was set to atmospheric and a zero velocity gradient in the direction normal to sliding was assumed. This can also be thought of as a fully developed flow approximation. The various parameter about cavity phenomenon (i.e. cavitation and no cavitation) is applied in the present work.

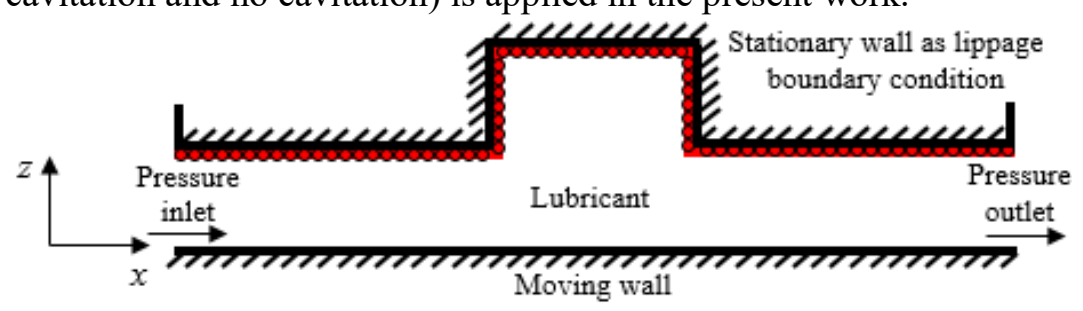

Figure 3. Slippage boundary condition of two-dimensional slider bearing.

\section{Results and discussions}

In this research, all of simulations were performed in the low inertia condition referring to the work of Dobrica and Fillon [1]. In this present study, the Reynolds number was set to 0.028, which means that the Navier-Stokes prediction and Reynolds equation have very close prediction [1]. This research is aimed to explore the inertia effect on the load support considering cavitation for slip and no slip situation.

Figure 4 shows the comparison between the Reynolds equation and the Navier-Stokes equation in terms of hydrodynamic pressure distribution varying texture length for the slip situation. It can be seen from Figure 4. that the deviation between the Reynolds equation and Navier-Stokes equation is not significant for all value of $b$ with and without cavitation model. However, when it is solved by NSE, there is a pressure jump in the edge of the texture cell. This prevails, for the case of cavitation and no cavitation. For the case of the bearing with low texture length (i.e $b=0.25 \mathrm{~mm}$ ), the pressure profile predicted by both Reynolds equation and Navier-Stokes equation with/without cavitation model is quite lower than that with higher $b$. However, for low $b 0.25 \mathrm{~mm}$, in this case, the cavitation region is also lowest. Based on the physical point of view it means the lowest $b$ can achieve the highest load support. In general speaking, for the bearing with low inertia, the Reynolds number is quite effective to solve the lubrication problem.

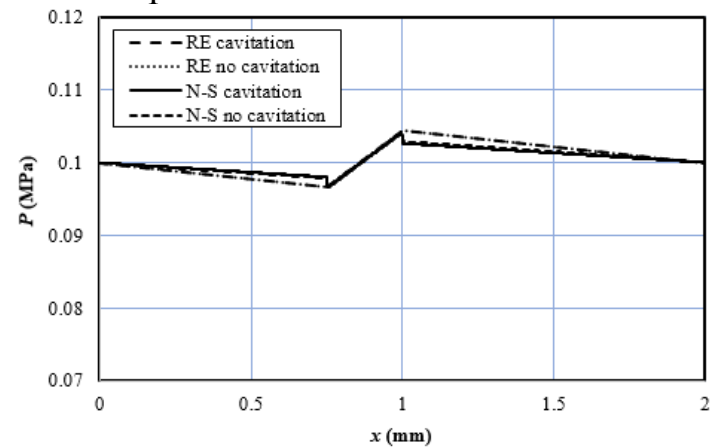

(a)

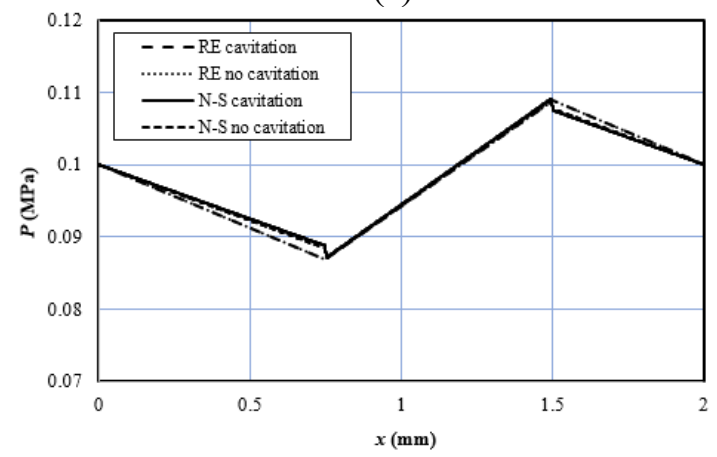

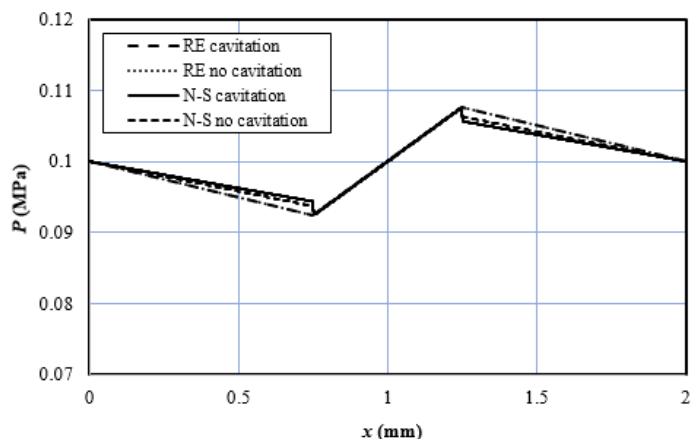

(b)

Figure 4. Pressure distribution in slip condition on texture length (a) $b=0.25 \mathrm{~mm}$, (b) $b=0.50$ $\mathrm{mm}$ and (c) $b=0.75 \mathrm{~mm}$. 
(c)

From Figure 5 it can be observed that prediction of RE and NSE in terms of hydrodynamic pressure are similar, it indicates that the inertia has no significant role in this pattern. Based on Figure 5 it can be seen that the increasing the texture length does not affect the cavitation effect. Based on the physical point of view it can be concluded that when the bearing is designed to have a low inertia effect, the cavitation effect does not change very much.

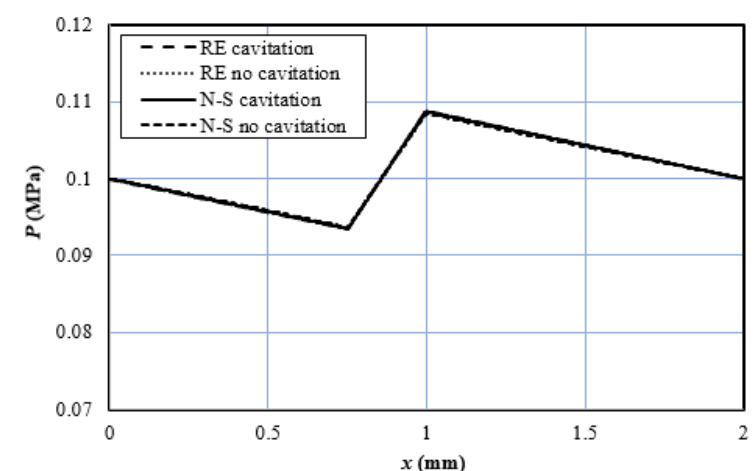

(a)

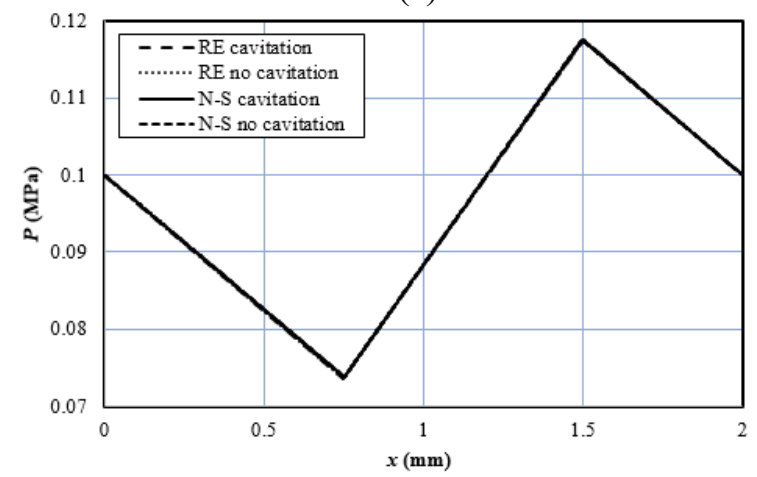

(c)

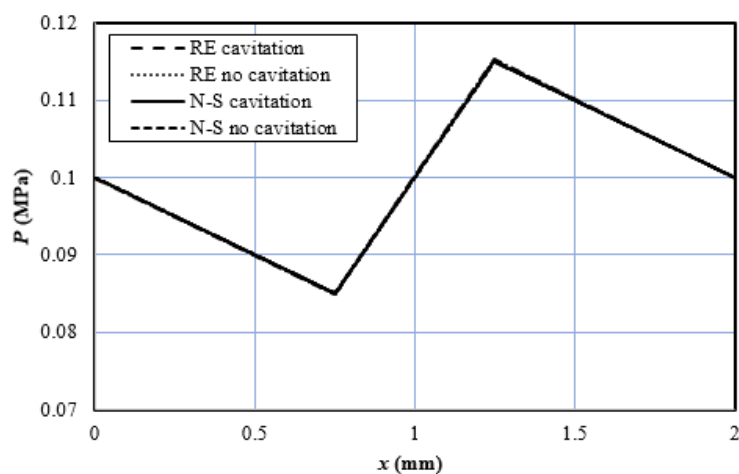

(b)

Figure 5. Pressure distribution in no-slip condition on texture length (a) $b=0.25 \mathrm{~mm}$, (b) $b=0.50 \mathrm{~mm}$ and (c) $b=0.75 \mathrm{~mm}$.

The inertia effect can be obtained by comparing the NSE and RE. Figure 4 shows that for the case of slip situation, the deviation between the Navier-Stokes and Reynolds equation is observed. Based on the pressure distribution between NSE and RE in slip conditions, there are differences at the peaks and valleys of the graph. But the difference is very small. It means that the slip condition can increase the inertia effect even though it is very small. Different results are shown in Figure 5 where no slip are applied. The pressure distribution looks the same between NSE and RE in all variations $b$ (texture length).

The most significant founding of this research is the fact that the slip has two influences on the load support: Slip can reduce the load support $W$ but at the sametime, slip can increase the load support. It can be observe from Table 1 that for low texture length $b$, the load support can be reduced by the slip up to $\pm 1 \%$. But for the case with high $b$, the load support can be improved by the slip $\pm 3 \%$. It seems that there is an "optimal value of texture length $b$ ". However, the investigation regarding to the optimal value of $b$ is out of the scope. 
Table 1. Load support of slider bearing.

\begin{tabular}{|c|c|c|c|c|c|c|}
\hline \multirow{3}{*}{ Parameters } & \multicolumn{6}{|c|}{ Load Support (Pa.m²) } \\
\hline & \multicolumn{3}{|c|}{ Reynolds Equation (RE) } & \multicolumn{3}{|c|}{ Navier-Stokes Equation (NSE) } \\
\hline & $b=0.25 \mathrm{~mm}$ & $b=0.50 \mathrm{~mm}$ & $b=0.75 \mathrm{~mm}$ & $b=0.25 \mathrm{~mm}$ & $b=0.50 \mathrm{~mm}$ & $b=0.75 \mathrm{~mm}$ \\
\hline $\begin{array}{l}\text { Slip and } \\
\text { cavitation }\end{array}$ & 201.107 & 200.000 & 195.598 & 200.655 & 199.996 & 196.256 \\
\hline $\begin{array}{l}\text { No-slip and } \\
\text { cavitation }\end{array}$ & 202.215 & 200.030 & 191.180 & 202.167 & 199.999 & 191.282 \\
\hline $\begin{array}{l}\text { Slip and no } \\
\text { cavitation }\end{array}$ & 201.107 & 200.000 & 195.598 & 200.738 & 199.995 & 196.127 \\
\hline $\begin{array}{l}\text { No-slip and no } \\
\text { cavitation }\end{array}$ & 202.215 & 200.030 & 191.180 & 202.083 & 199.999 & 191.262 \\
\hline
\end{tabular}

\section{Conclusions}

The hydrodynamic pressure in single textured bearing with slip and no slip condition, considering and not considering cavitation has solved by the modified Reynold equation (RE) and Navier-Stokes (N-S) solution. The effect of texture length variation is of particular interest. Based on the results and discussion, the following conclusion can be drawn as follows:

1. Increasing the inertia effect (i.e lowering texture length $b$ ) can increase the load support for all situation (slip or no slip, cavitation or no cavitation),

2. Slip can reduce the load support, but on the other hand slip also can increase the load support depending on the texture length,

3. The cavitation effects is not found in the bearing pattern in which the inertia is not significant.

\section{References}

[1] Dobrica M B and Fillon M 2009 Proc. Instn. Mech. Eng. Part J: J. of Eng. Tribol 223(1) 69-78

[2] Armentrout R W, He M, Haykin T and Reed A E 2017 Tribol. Trans 60(6) 1129-47

[3] Remy B, Bou-Saïd B and Lamquin T 2016 Tribol. Int 95 139-46

[4] Syed I and Sarangi M 2014 Tribol. Int 69 30-8.

[5] Woloszynski T, Podsiadlo P and Stachowiak G W 2015 Tribol. Int 91 170-6

[6] Dousti S, Allaire P, Dimond T and Cao J 2016 Tribol. Int 102 182-97.

[7] Cupillard S, Glavatskih S and Cervantes M J 2010 Proc. Instn. Mech. Eng. Part J: J. of Eng. Tribol 224(8) 751-56

[8] Okabe E P 2017 Tribol. Int 114 245-56.

[9] Fowell M, Olver A V, Gosman A D, Spikes H A and Pegg I 2007 J. of Tribol 129(2) 336-47.

[10] Shi X and Ni T 2011 Tribol. Int 44(12) 2022-28

[11] Rao T V V L N, Rani A M A, Nagarajan T and Hashim F M 2012 Tribol. Int 56 121-8

[12] Zhang H, Hua M, Dong G N, Zhang D Y and Chin K S 2014 Tribol. Int 79 32-41

[13] Tauviqirrahman M, Muchammad, Jamari and Schipper D J 2014 Tribol. Trans 57(1) 134-45

[14] Cheng F and Ji W 2016 Tribol. Int 97 163-72

[15] Zhang W M, Meng G and Wei K X 2012 Tribol. Trans 55(1) 71-6

[16] Muchammad M, Tauviqirrahman M, Jamari J and Schipper D J 2017 Lubr. Sci 29(3) 133-52

[17] ANSYS, ANSYS Fluent, version 12.0: theory guide. ANSYS, Inc., Canonsburg, USA., 2009. 